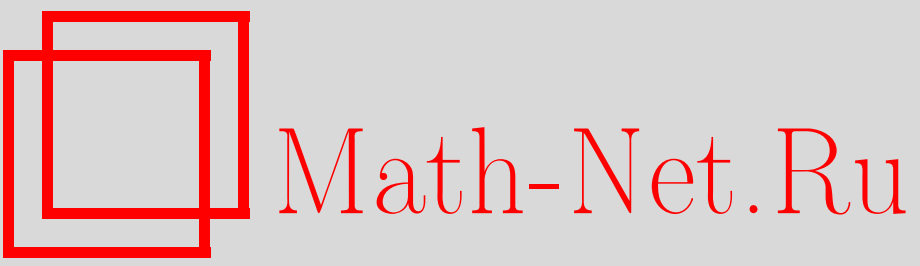

Т. Р. Арутюнян, Двусторонний метод расчета магнитостатического поля, Итоги науки и техн. Сер. Соврем. мат. и ее прил. Темат. обз., 2021, том 191, 3-9

DOI: https://doi.org/10.36535/0233-6723-2021-191-3-9

Использование Общероссийского математического портала Math-Net.Ru подразумевает, что вы прочитали и согласны с пользовательским соглашением

http://www.mathnet.ru/rus/agreement

Параметры загрузки:

IP : 54.197 .217 .227

26 апреля 2023 г., $16: 41: 43$ 
ИТОГИ НАУКИ И ТЕХНИКИ.

Современная математика и ее приложения.

Тематические обзоры.

Том 191 (2021). С. 3-9

DOI: 10.36535/0233-6723-2021-191-3-9

УДК 519.63

\title{
ДВУСТОРОННИЙ МЕТОД РАСЧЕТА МАГНИТОСТАТИЧЕСКОГО ПОЛЯ
}

\author{
(C) 2021 г. $\quad$ T. Р. АРУТЮНЯН
}

\begin{abstract}
АннотАция. Рассмотрены двусторонние методы математического моделирования магнитного поля электротехнических систем, содержащих ферромагнетики и постоянные магниты. Методы основаны на применении к уравнениям электромагнитного поля в терминах скалярного потенциала метода множителей Лагранжа.
\end{abstract}

Ключевъе слова: магнитное поле, двусторонний метод, погрешность коэффициентов, ферромагнетик, постоянный магнит, магнитный потенциал, метод множителей Лагранжа.

\section{BILATERAL METHOD OF CALCULATING MAGNETOSTATIC FIELDS}

\author{
(C) 2021 T. R. ARUTYUNYAN
}

\begin{abstract}
Bilateral methods of mathematical modeling of magnetic fields in electrical systems containing ferromagnets and permanent magnets are considered. The methods are based on the Lagrange multiplier method applied to the equations of the electromagnetic field in terms of the scalar potential.
\end{abstract}

Keywords and phrases: magnetic field, bilateral method, coefficient error, ferromagnetic material, permanent magnet, magnetic potential, Lagrange multiplier method.

AMS Subject Classification: $78 \mathrm{Mxx}$

1. Введение. Математическое моделирование технических систем с учетом неустранимой погрешности является одной из актуальных научных проблем. Традиционным методом, широко применяемым для учета погрешности коэффициентов математической модели, является метод малого параметра. Недостатки этого метода связаны с тем, что он является асимптотическим и недостаточно надежным, так как его точность существенно зависит от величины погрешности исходных данных. Другим подходом является применение методов интервального анализа и двусторонних методов. Однако соответствующие методы разработаны преимущественно для систем с сосредоточенными параметрами. В статье развивается соответствующий вариационный подход на случай распределенных магнитостатических систем. Вместо принципа максимума Понтрягина используется более общий и практичный метод Лагранжа, применимый как для динамических, так и стационарных задач. Преимущества предложенного метода наибольшие при учете многомерной функциональной погрешности. В статье в качестве примера рассматривается магнитная система, состоящая из конструкционных частей из ферромагнитных материалов с известными характеристиками. Одна из актуальных проблем теории расчета магнитного поля таких устройств связана с учетом влияния ширины петли магнитного гистерезиса материала на точность расчета магнитного поля. Учет в модели остаточной намагниченности позволяет рассчитывать системы с постоянными магнитами (см. [1-3]). 


\section{2. Основные соотношения метода.}

2.1. Формулировка краевой задачи. Рассмотрим задачу расчета параметров и характеристик стационарного магнитного поля в некоторой магнитной системе (см. [1-3]). Предположим, что материал с магнитными свойствами (вообще говоря, неоднородный с нелинейными и анизотропными свойствами) занимает объем $V$. Намагниченность связана с индукцией и напряженностью магнитного поля согласно векторному соотношению: $\boldsymbol{M}=\boldsymbol{B} / \mu_{0}-\boldsymbol{H}$. В немагнитной среде $\boldsymbol{B}=\mu_{0} \boldsymbol{H}$, в ферромагнетике $\boldsymbol{B}=\mu_{0}(\boldsymbol{H}+\boldsymbol{M})$. Для моделирования поля в постоянном магните требуется учитывать остаточную намагниченность $\boldsymbol{M}_{n}$ (см. [3]): $\boldsymbol{M}=\chi \boldsymbol{H}+\boldsymbol{M}_{n}$, где $\chi=\mu / \mu_{0}-1$ - магнитная восприимчивость материала, $\mu$ - удельная магнитная проницаемость определяется основной кривой намагничивания $\mu=\mu(\boldsymbol{B})$, в воздухе, изоляционных материалах и шинах $\mu=\mu_{0}, \Gamma_{\mathrm{H}} / \mathrm{м}$. Для стационарного поля определяется скалярный магнитный потенциал: $\boldsymbol{H}=-\nabla \phi$. Предполагаем, что на бесконечности потенциал равен нулю. Сформулируем краевую задачу для расчета стационарного магнитного поля в пространстве. Будем предполагать, что магнитная проницаемость объема ферромагнитного материала на несколько порядков выше магнитной проницаемости воздуха. Тогда краевая задача расчета поля в пространстве может быть разбита на две: в воздухе решается уравнение Лапласа для магнитного потенциала, при нулевом краевом условии на границе ферромагнетика и заданных источниках поля в пространстве: $\boldsymbol{H}=\boldsymbol{H}_{\mathrm{ex}}-\nabla v_{\mathrm{ex}}$, $\boldsymbol{H}_{\mathrm{ex}}=-\nabla v_{\mathrm{ex}}$

$$
\Delta V=0,(x, y) \in C(D+\Gamma) ; \quad v=-v_{\mathrm{ex}}(x, y) \in \Gamma=\partial D,
$$

В объеме ферромагнитного тела или постоянного магнита решается краевая задача второго типа для магнитного потенциала при заданной плотности магнитного потока сквозь границу объема:

$$
\begin{gathered}
\frac{\partial}{\partial x}\left(\mu(H) \frac{\partial u}{\partial x}\right)+\frac{\partial}{\partial y}\left(\mu(H) \frac{\partial u}{\partial y}\right)=0, \quad(x, y) \in D ; \\
\mu(H) \frac{\partial u}{\partial n}=\mu_{0} \frac{\partial v}{\partial n}, \quad(x, y) \in \Gamma ; \\
u(0,0)=0, \quad \boldsymbol{H}=-\nabla u .
\end{gathered}
$$

Требуется решить записанные уравнения с учетом погрешности задания кривой намагничивания:

$$
\mu=\mu(H) \in\left(\mu^{-}(H), \mu^{+}(H)\right), \quad w_{0}=\mu^{+}(H)-\mu^{-}(H) .
$$

Предположим, требуется найти оценку решения краевой задачи снизу (сверху):

$$
u\left(x_{0}, y_{0}\right) \rightarrow \min (\max ), \quad \mu=\mu(H) \in\left(\mu^{-}(H), \quad \mu^{+}(H)\right),
$$

Функционал Лагранжа рассматриваемой задачи имеет вид:

$$
\begin{aligned}
L=\iint_{D} w\left(x_{N}, y_{N}\right) & \left(\frac{\partial}{\partial x_{N}}\left(\mu\left(\left|\nabla_{N} u\right|\right) \frac{\partial u}{\partial x_{N}}\right)+\frac{\partial}{\partial y_{N}}\left(\mu\left(\left|\nabla_{N} u\right|\right) \frac{\partial u}{\partial y_{N}}\right)\right) d x_{N} d y_{N}+ \\
+\int_{\Gamma} w_{1}\left(x_{N}, y_{N}\right) & \left(\mu\left(\left|\nabla_{N} u\right|\right) \frac{\partial u}{\partial n_{N}}-\mu_{0} \frac{\partial v}{\partial n_{N}}\right) d \Gamma_{N}+w_{2} u(0,0)+ \\
& +\iint_{D} u\left(x_{N}, y_{N}\right) \delta\left(x_{N}-x_{0}, y_{N}-y_{0}\right) d x_{N} d y_{N}=0, \quad(x, y) \in D .
\end{aligned}
$$

Первый интеграл в данном функционале преобразуется к виду

$$
\int_{\Gamma} w\left(x_{N}, y_{N}\right) \mu_{0} \frac{\partial v}{\partial n_{N}} d \Gamma_{N}-\iint_{D} \mu\left(\left|\nabla_{N} u\right|\right) \nabla_{N} u \nabla_{N} w d x_{N} d y_{N}=0 .
$$

Для решения задачи согласно известной методике вычисляется вариация по функции $u$ и находится уравнение экстремалей данного функционала (см. $[4,5,7,8])$. Соответствующие верхней (нижней) оценке решения значения находятся из условия экстремума функционала Лагранжа 
$L \rightarrow \max (\min )$ для всех $\mu(|\nabla u|)$ из допустимого множества (5). Экстремальное значение достигается, если магнитная характеристика описывается выражением

$$
\mu^{*}=\mu^{0}(|\nabla u|)(1-0,5 \omega(|\nabla u|) \operatorname{sign}(\nabla u \nabla p)) .
$$

Сопряженное уравнение имеет вид

$$
\operatorname{div}\left(\mu^{*}(|\nabla u|) \nabla p+H^{-1} \frac{d \mu^{*}}{d H}(\nabla u \nabla p) \nabla u\right)+\delta\left(x-x_{0}, y-y_{0}\right)-\delta(x, y)=0, \quad(x, y) \in D .
$$

Если требуется оценить не равномерную, а среднеквадратическую погрешность, например,

$$
\left\|u-u^{0}\right\|_{L_{2}(D)} \rightarrow \min (\max ),
$$

то сопряженное уравнение имеет вид:

$$
\operatorname{div}\left(\mu^{*}(|\nabla u|) \nabla p+H^{-1} \frac{d \mu^{*}}{d H}(\nabla u \nabla p) \nabla u\right)+C_{0}\left(u-u^{0}\right)=0, \quad(x, y) \in D .
$$

2.2. Конечно-разностный метод решения краевой задачи. Система МКР на неравномерной сетке, аппроксимирующая краевую задачу (1), имеет следующий вид:

$$
\begin{gathered}
\frac{1}{h_{x, i}^{0}}\left(\frac{v_{i+1, j}-v_{i, j}}{h_{x, i+1}}-\frac{v_{i, j}-v_{i-1, j}}{h_{x, i}}\right)+\frac{1}{h_{y, i}^{0}}\left(\frac{v_{i, j+1}-v_{i, j}}{h_{y, j+1}}-\frac{v_{i, j}-v_{i, j-1}}{h_{y, j}}\right)=0, \\
(i, j) \in C D_{h}, \quad D_{h}=\left((i, j) \mid i=-N_{x}, \ldots, N_{x} ; j=-N_{y}, \ldots, N_{y}\right) .
\end{gathered}
$$

Аналогичная система MKР для нелинейной краевой задачи (2)-(4) с переменным коэффициентом:

$$
\begin{aligned}
\frac{1}{h_{x, i}^{0}}\left(a_{i+1 / 2, j} \frac{u_{i+1, j}-u_{i, j}}{h_{x, i+1}}\right. & \left.-a_{i-1 / 2, j} \frac{u_{i, j}-u_{i-1, j}}{h_{x, i}}\right)+ \\
& +\frac{1}{h_{y, i}^{0}}\left(a_{i, j+1 / 2} \frac{u_{i, j+1}-u_{i, j}}{h_{y, j+1}}-a_{i, j-1 / 2} \frac{u_{i, j}-u_{i, j-1}}{h_{y, j}}\right)=0, \quad(i, j) \in D_{h},
\end{aligned}
$$

где $h_{x, i}, h_{y, j}$ - шаги сетки по координатам $x$ и $y ; i, j$-номера узла сетки по пространственным координатам $x$ и $y$;

$$
\begin{gathered}
h_{x, i}^{0}=\frac{h_{x, i}+h_{x, i+1}}{2}, \quad h_{y, j}^{0}=\frac{h_{y, j}+h_{y, j+1}}{2}, \\
u_{i, j} \approx u\left(x_{i}, y_{j}\right), \quad v_{i, j} \approx v\left(x_{i}, y_{j}\right), \quad(i, j) \in D_{h}, \\
x_{i}=i h, \quad y_{j}=j h, \quad v_{i,-N_{y}}=M_{0} y_{-N_{y}}, \quad v_{i, N_{y}}=M_{0} y_{N_{y}}, \quad M_{0}=B_{r} / \mu_{0},
\end{gathered}
$$

значения коэффициентов системы МКР, отвечающие максимуму решения:

$$
a_{i, j}=\mu\left(H_{i, j}\right)\left(1-0,5 w_{a} \cdot \operatorname{sign}\left(H_{x, i, j} w_{x, i, j}+H_{y, i, j} w_{y, i, j}\right)\right),
$$

где $w_{a}$ - относительная погрешность характеристики намагничивания. Уравнения системы МКР приводятся к виду, удобному для применения итерационного метода релаксации и Зейделя. Для внутренних узлов соответствующих областей:

$$
\begin{gathered}
v_{i, j}=\left(v_{i-1, j}+v_{i+1, j}+v_{i, j-1}+v_{i, j+1}\right) / 4 \\
u_{i, j}=\frac{\left(a_{i, j}+a_{i-1, j}\right) u_{i-1, j}+\left(a_{i, j}+a_{i+1, j}\right) u_{i+1, j}+\left(a_{i, j}+a_{i, j-1}\right) u_{i, j-1}+\left(a_{i, j}+a_{i, j+1}\right) u_{i, j+1}}{a_{i-1, j}+a_{i+1, j}+a_{i, j-1}+a_{i, j+1}+4 a_{i, j}} .
\end{gathered}
$$

Конечно-разностные соотношения, соответствующие краевым условиям:

$$
\begin{array}{lll}
i=-N_{x} ; & u_{i, j}=u_{i+1, j}-\mu_{0}\left(v_{i, j}-v_{i-1, j}\right) / a_{i, j}, & j=-N_{y}, \ldots, N_{y} ; \\
i=N_{x} ; & u_{i, j}=u_{i-1, j}+\mu_{0}\left(v_{i+1, j}-v_{i, j}\right) / a_{i, j}, & j=-N_{y}, \ldots, N_{y} ; \\
j=-N_{y} ; & u_{i, j}=u_{i, j+1}-\mu_{0}\left(v_{i, j}-v_{i, j-1}\right) / a_{i, j}, & i=-N_{x}, \ldots, N_{x} ; \\
i=N_{y} ; & u_{i, j}=u_{i, j-1}+\mu_{0}\left(v_{i, j}-v_{i, j-1}\right) / a_{i, j}, & i=-N_{x}, \ldots, N_{x} .
\end{array}
$$


Сеточные компоненты вектора напряженности во внутренних узлах вычисляются при помощи центральных разностей:

$$
H_{x, i, j}=-\frac{u_{i+1, j}-u_{i-1, j}}{2 h_{x, i}^{0}}, \quad H_{y, i, j}=-\frac{u_{i, j+1}-u_{i, j-1}}{2 h_{y, j}^{0}} .
$$

Значения магнитной индукции в узлах сетки:

$$
B_{x, i, j}=a_{i, j} H_{x, i, j}, \quad B_{y, i, j}=a_{i, j} H_{y, i, j} .
$$

Рассмотрим соотношения МКР для сопряженной краевой задачи. Итерации осуществляются при помощи методов Зейделя и релаксации:

$$
\begin{gathered}
R_{1}=\left(a_{i, j}^{(1)}+a_{i-1, j}^{(1)}\right) w_{i-1, j}+\left(a_{i, j}^{(1)}+a_{i+1, j}^{(1)}\right) w_{i+1, j}+\left(a_{i, j}^{(2)}+a_{i, j-1}^{(2)}\right) w_{i, j-1}+ \\
+\left(a_{i, j}^{(2)}+a_{i, j+1}^{(2)}\right) w_{i, j+1}+\left(a_{i-1, j}^{(3)}+a_{i, j-1}^{(3)}\right) w_{i-1, j-1}+\left(-a_{i-1, j}^{(3)}-a_{i, j+1}^{(3)}\right) w_{i-1, j+1}+ \\
+\left(-a_{i+1, j}^{(3)}-a_{i, j-1}^{(3)}\right) w_{i+1, j-1}+\left(a_{i+1, j}^{(3)}+a_{i, j+1}^{(3)}\right) w_{i+1, j+1}+ \\
+h_{x, i, j} h_{y, i, j}\left(d\left(x_{i}-x_{0}, y_{j}-y_{0}\right)-d\left(x_{i}, y_{j}\right)\right), \\
R_{2}=a_{i-1, j}^{(1)}+a_{i+1, j}^{(1)}+a_{i, j-1}^{(2)}+a_{i, j+1}^{(2)}+2 a_{i, j}^{(1)}+2 a_{i, j}^{(2)} ; \\
w_{i, j}^{(1)}=R_{1} / R_{2},
\end{gathered}
$$

где $r$ - параметр релаксации. Значения коэффициентов системы МКР вычисляются по формулам

$$
\begin{gathered}
d a_{i, j}=d \mu / d H\left(\sqrt{H_{x, i, j}^{2}+H_{y, i, j}^{2}}\right) ; \\
a_{i, j}^{(1)}=a_{i, j}+d a_{i, j} H_{x, i, j}^{2} / \sqrt{H_{x, i, j}^{2}+H_{y, i, j}^{2}} ; \\
a_{i, j}^{(2)}=a_{i, j}+d a_{i, j} H_{y, i, j}^{2} / \sqrt{H_{x, i, j}^{2}+H_{y, i, j}^{2}} ; \\
a_{i, j}^{(3)}=a_{i, j}+d a_{i, j} H_{x, i, j} H_{y, i, j} / \sqrt{H_{x, i, j}^{2}+H_{y, i, j}^{2}} .
\end{gathered}
$$

Соотношения для краевых условий:

$$
\begin{gathered}
i=-N_{x} ; \quad w_{i, j}=w_{i+1, j}-\frac{h_{x, i} d a_{i, j} H_{y, i, j} w_{y, i, j}}{a_{i, j}^{(1)} \sqrt{H_{x, i, j}^{2}+H_{y, i, j}^{2}}}, \quad j=-N_{y}, \ldots, N_{y} ; \\
i=N_{x} ; \quad w_{i, j}=w_{i-1, j}-\frac{h_{x, i} d a_{i, j} H_{y, i, j} w_{y, i, j}}{a_{i, j}^{(1)} \sqrt{H_{x, i, j}^{2}+H_{y, i, j}^{2}}}, \quad j=-N_{y}, \ldots, N_{y} ; \\
j=-N_{y} ; \quad w_{i, j}=w_{i, j+1}-\frac{h_{y, i} d a_{i, j} H_{x, i, j} w_{x, i, j}}{a_{i, j}^{(2)} \sqrt{H_{x, i, j}^{2}+H_{y, i, j}^{2}}}, \quad i=-N_{x}, \ldots, N_{x} ; \\
j=N_{y} ; \quad w_{i, j}=w_{i, j-1}-\frac{h_{y, i} d a_{i, j} H_{x, i, j} w_{x, i, j}}{a_{i, j}^{(2)} \sqrt{H_{x, i, j}^{2}+H_{y, i, j}^{2}}}, \quad i=-N_{x}, \ldots, N_{x} ;
\end{gathered}
$$

где компоненты градиента решения сопряженной краевой задачи вычисляются при помощи формул центральных разностей:

$$
w_{x, i, j}=-\frac{w_{i+1, j}-w_{i-1, j}}{2 h_{x, i}^{0}}, w_{y, i, j}=-\frac{w_{i, j+1}-w_{i, j-1}}{2 h_{y, i}^{0}} .
$$

Системы МКР решаются итерационно (вначале решаются системы, соответствующие основным уравнениям поля (2)-(4), затем решается краевая задача для сопряженного уравнения и далее попеременно). Итерации оканчиваются, если имеет место совпадение требуемого количества знаков в числовых значениях приближений. Примером альтернативного метода решения является подход на основе интервальных оценок (см. [6]). 


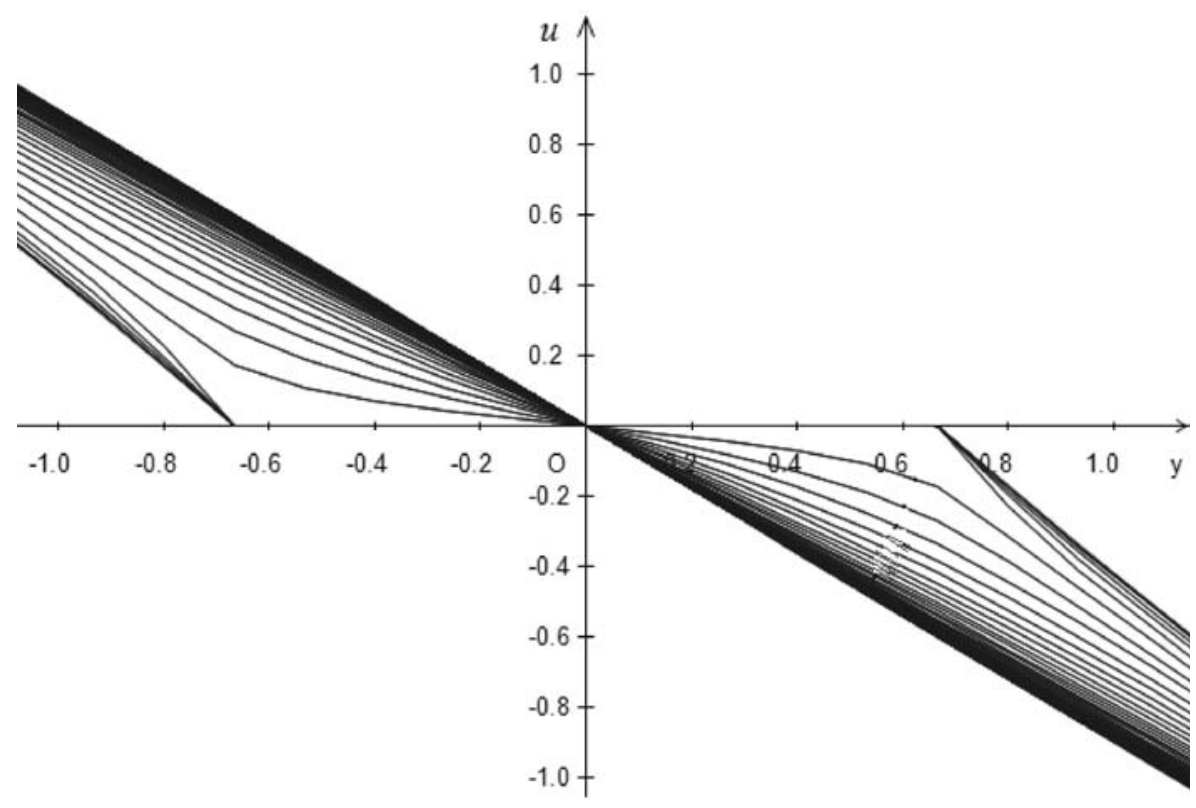

Рис. 1. Графики зависимости магнитного потенциала в воздухе от ординаты при фиксированных значениях абсциссы (масштабы $1: 7,5$ и $1: 2 / 3$ )

3. Модельная задача двусторонней оценки решения уравнений магнитостатики в объеме ферромагнита. Рассмотрим случай ферромагнетика призматической формы с квадратным сечением:

$$
\begin{array}{lll}
\frac{d M_{f}}{d H}=M_{s}\left(-\mathrm{sh}^{-2} \frac{H}{a}+a H^{-2}\right), & \frac{d M_{f}}{d H}(0)=\frac{M_{s}}{3 a}, \\
\frac{d \mu}{d H}=\mu_{0}\left(\frac{d M_{f}}{d H}-\frac{M_{f}(H)}{H}\right), & \frac{d \mu}{d H}(0)=0 .
\end{array}
$$

В начальный момент времени появляется равномерное стороннее магнитное поле с индукцией $B_{0}=0,8$ Тл, направленное вдоль оси $O Y$, индуцирующее поле в ферромагнетике. Требуется найти двусторонние оценки решения в заданной точке пространства. В расчетах размер стороны квадрата принят равным единице. $L_{x}=1, L_{y}=1$. Количество разбиений по осям координат области ферромагнетика: $n_{x}, n_{y}=5$. Размеры ячеек $h_{x}=L_{x} / n_{x}, h_{y}=L_{y} / n_{y}$. Размер расчетной области был выбран в 5 раз больше при тех же шагах сетки. Основная кривая намагничивания вычисляется по формуле Ланжевена $M_{f}(H)=M_{s}(\operatorname{cth}(H / a)-a / H)$, параметры $M s$, $a$ определяются материалом. Значения параметров для электротехнической стали марки Э330A равны $M s=1648136,0 ; a=55,2$. Погрешность задания кривой намагничивания в расчетах принималась равной 10-20\%. Результаты вычисления равномерных оценок решения сверху и снизу даны в таблице 1 . Точность решения сеточных уравнений порядка $10^{-4} \ldots 10^{-5}$. Решение находилось итерационным методом релаксации (коэффициент релаксации 0,1 ).

Как показывают результаты решения краевой задачи в таблице 1, для всех рассмотренных точек области сохранилось свойство двусторонности оценок.

На рис. 1 и 2 показаны графики, характеризующие магнитное поле в воздухе и в области ферромагнетика. Асимметрия графиков на рис. 2 связана с особенностью краевой задачи - находятся оценки сверху (снизу), что влияет на симметрию решения задачи. На рис. 3 даны графики решения сопряженной краевой задачи в относительных координатах (графики нормированы на величину, кратную среднему абсолютному значению по области).

4. Заключение. Описанный метод предоставляет удобные возможности для нахождения оценок решения в окрестности особых точек, либо для оценки среднеквадратической погрешности 
Таблица 1. Результаты решения краевой задачи

\begin{tabular}{|c|c|r|r|r|c|}
\hline$\langle|p|\rangle$ & $\max |p|$ & $w_{0}(A)$ & $x_{0}$ & $y_{0}$ & $A\left(x_{0}, y_{0}, t_{F}\right)$ \\
\hline 1964,85007 & 1988,31583 & $-0,10$ & -5 & -5 & 262,79312 \\
\hline 2043,21236 & 2066,63434 & 0,10 & -5 & -5 & 647,81461 \\
\hline 4614,15949 & 4641,52274 & $-0,10$ & -4 & -4 & 169,43313 \\
\hline 4126,44905 & 4152,71996 & 0,10 & -4 & -4 & 333,67402 \\
\hline 4806,65968 & 4835,36997 & $-0,10$ & -3 & -3 & 121,60234 \\
\hline 4303,07974 & 4344,20867 & 0,10 & -3 & -3 & 223,87075 \\
\hline 4897,80009 & 4925,72391 & $-0,10$ & -2 & -2 & 76,25577 \\
\hline 4473,04123 & 4521,28807 & 0,10 & -2 & -2 & 140,02441 \\
\hline 4960,69039 & 4980,30642 & $-0,10$ & -1 & -1 & 35,35703 \\
\hline 5155,11358 & 5202,20330 & 0,10 & -1 & -1 & 76,23501 \\
\hline 5050,67244 & 5060,37790 & $-0,10$ & 0 & 0 & 0 \\
\hline 5256,78002 & 5286,14070 & 0,10 & 0 & 0 & 0 \\
\hline 5083,89098 & 5094,31434 & $-0,10$ & 1 & 1 & $-47,8860$ \\
\hline 5346,43769 & 5367,48972 & 0,10 & 1 & 1 & $-53,39003$ \\
\hline 5196,13915 & 5216,94888 & $-0,10$ & 2 & 2 & $-105,93750$ \\
\hline 5506,17150 & 5532,85193 & 0,10 & 2 & 2 & $-93,66607$ \\
\hline 5270,42920 & 5297,91971 & $-0,10$ & 3 & 3 & $-145,01243$ \\
\hline 5615,00142 & 5646,95074 & 0,10 & 3 & 3 & $-118,71798$ \\
\hline 5426,90117 & 5455,84568 & $-0,10$ & 4 & 4 & $-336,16334$ \\
\hline 5694,49215 & 5720,20326 & 0,10 & 4 & 4 & $-173,69809$ \\
\hline 5501,54560 & 5525,75066 & $-0,10$ & 5 & 5 & $-647,81466$ \\
\hline 5788,50031 & 5812,24166 & 0,10 & 5 & 5 & $-262,79313$ \\
\hline
\end{tabular}

решения во всей области. Примерами соответствующих актуальных научно-технических задач являются оценки коммутационных перенапряжений, бросков тока при включении цепей с нелинейными индуктивностями, прямые и обратные задачи расчета постоянных магнитов, при оценке влияния ширины петли гистерезиса. Рассмотренный метод может применяться при решении как стационарных, так и динамических задач с учетом погрешности коэффициентов. Предложенный метод может применяться в целях повышения надежности результатов в проектных расчетах различных электротехнических устройств, а также в задачах теории магнитных измерений.

\section{СПИСОК ЛИТЕРАТУРЫ}

1. Арутюнян Р. В., Некрасов С. А., Середина П. Б. Идентификация намагниченности постоянных магнитов на основе метода скалярного магнитного потенциала// Изв. вузов. Электромех. $-2018 .-61$, № 6. - C. 19-25.

2. Арутюнян Р. В., Некрасов С. А., Середина П. Б. Методы идентификации намагниченности постоянных магнитов на основе интегральных уравнений. Исследование и примеры применения// Изв. вузов. Электромех. - 2019. - 1.

3. Блох Ю. И. Теоретические основы комплексной магниторазведки. - М.: МГГА, 2012.

4. Василъев Ф. П. Численные методы решения экстремальных задач. - М.: Наука, 1988.

5. Корн Г., Корн Т. Справочник по математике. - М.: Наука, 1978.

6. Рогалев A. Н. Границы множеств решений систем обыкновенных дифференциальных уравнений с интервальными начальными данными// Вычисл. технол. - 2004. - 9, № 1. - С. 86-94. 


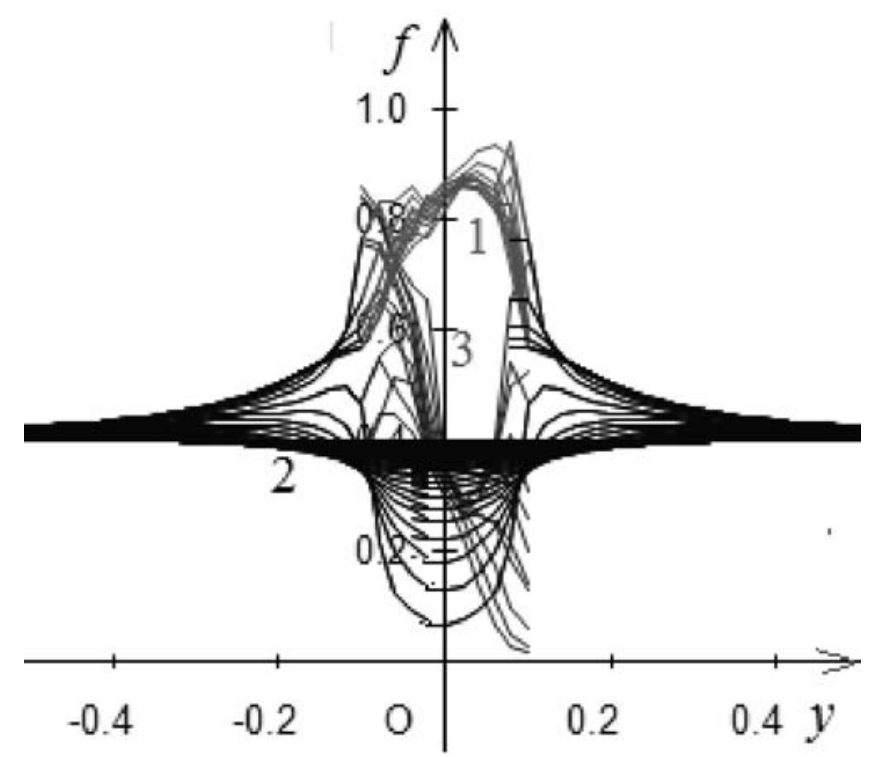

Рис. 2. Графики магнитной индукции после 10 итераций в магнетике (1), в воздухе (2) и удельная магнитная проводимость (3) (масштабы для серии графиков (1) и (2) $1: 2$, для серии графиков $(3)-1: \mu(0)), \omega=-0,1$ э

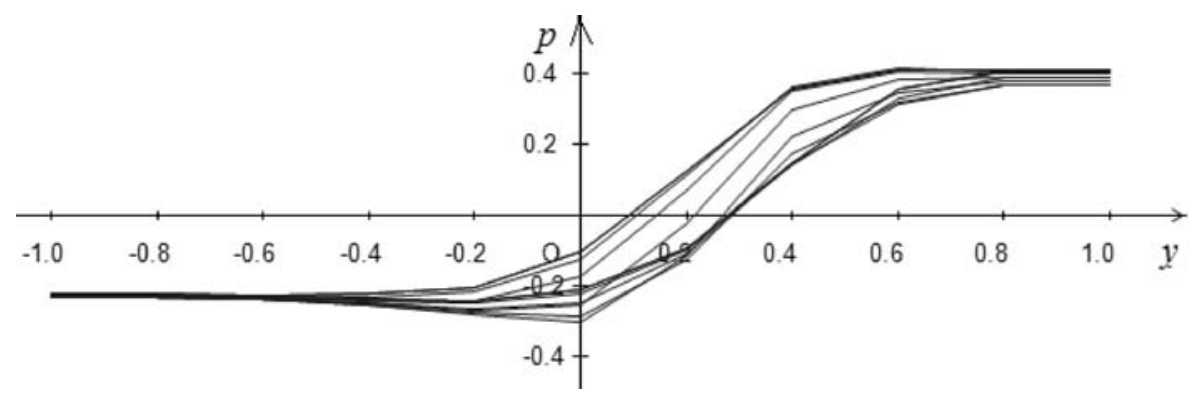

Рис. 3. Графики решения сопряженной краевой задачи после 10 итераций (в относительных координатах)

7. Справочник по автоматическому управлению. - М.: Наука, 1987.

8. Треногин В. А. Функциональный анализ. - М.: Наука, 1980.

Арутюнян Тигран Робертович

Московский технический университет связи и информатики

E-mail: tigran_201094@mail.ru 